\title{
Downscaling of coarse-resolution radiometer brightness temperature by high-resolution radar backscatter
}

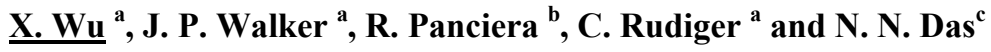 \\ ${ }^{a}$ Department of Civil Engineering, Monash University, Clayton, VIC 3800, Australia \\ ${ }^{b}$ Cooperative Research Centre for Spatial Information, Melbourne, VIC 3053, Australia \\ ${ }^{c}$ Jet Propulsion Laboratory (NASA), California Institute of Technology, California 91109, USA \\ Email: xiaoling.wu@monash.edu
}

\begin{abstract}
Given the importance of soil moisture for hydrological applications, such as weather and flood forecasting, passive microwave remote sensing is a promising approach for retrieving soil moisture due to its high sensitivity to near-surface soil moisture, applicability to all weather conditions, direct relationship with the soil dielectric constant, and reduced effects from vegetation and roughness. However, passive microwave (radiometer) observations suffer from being relatively low spatial resolution, on the order of $36 \mathrm{~km}$. It is proposed that this scale issue may be overcome by using active microwave (radar) observations, which have much higher resolution when using Synthetic Aperture Radar (SAR) techniques $(<3 \mathrm{~km})$, and this is the approach being taken by NASA's Soil Moisture Active Passive (SMAP) mission, with a scheduled launch in late 2014. The rationale behind SMAP is to use the synergy between active and passive observations in a downscaling approach to overcome the individual limitations of each observation type, and ultimately provide a merged soil moisture data set at intermediate resolution $(\sim 9 \mathrm{~km})$.
\end{abstract}

The objective of this study is to test the proposed baseline downscaling approach for the SMAP mission using airborne data, thus assessing its viability for future application to SMAP data. The approach is based on the hypothesis of a near-linear relationship between radiometer brightness temperature $(T b)$ and SAR backscatter $(\sigma)$, and has thus far received very limited testing. The experimental dataset used in this study was collected during the Soil Moisture Active Passive Experiment (SMAPEx) field campaigns over a study site in south-eastern Australia approximately $38 \mathrm{~km} \times 36 \mathrm{~km}$ in size, equivalent to a SMAP radiometer pixel. This research focuses on the brightness temperature downscaling algorithm; according to the SMAP Algorithm Theoretical Basis Documents these downscaled brightness temperatures will subsequently be converted to soil moisture at fine resolution through the traditional passive microwave retrieval algorithm.

The baseline downscaling algorithm was applied to high resolution data from SMAPEx, which include $1 \mathrm{~km}$ resolution brightness temperature collected by the Polarimetric L-band Multibeam Radiometer (PLMR) and $\sim 10 \mathrm{~m}$ resolution backscatters collected by the Polarimetric L-band Imaging Synthetic aperture radar (PLIS). To minimize noise in the radar data and to approximate the SMAP radiometer/radar pixel ratios $(36 \mathrm{~km} T b$ to $9 \mathrm{~km}$ resolution using $3 \mathrm{~km} \sigma$ ) the PLIS data were aggregated to $250 \mathrm{~m}$ resolution, so as to downscale $1 \mathrm{~km} T b$ to $250 \mathrm{~m}$ resolution, thus keeping the same ratio of radiometer/SAR spatial resolution as the SMAP mission. Results showed that the Root-Mean-Square Error (RMSE) in $T b$ downscaled at $100 \mathrm{~m}$ resolution was around $10 K$ at $h$-polarization and $8 K$ at $v$-polarization over a cropping area. This RMSE was reduced to $9 K$ and $7 K$ respectively when downscaling to $250 \mathrm{~m}$ resolution, due to a decreased spatial heterogeneity during averaging. It was also noted that results at $v$-polarization were slightly better than those at $h$-polarization, since the backscatter is more linearly related to $T b$ at $v$-polarization than $T b$ at $h$-polarization. The accuracy of the downscaling over grassland sites was improved by approximately $3 K$ with respect to the cropping area. This was attributed to the more heterogeneous conditions in cropping areas, compared to the relatively uniform conditions in the grassland area. However, one limitation of this study was the availability of only three days of data for estimating the linearity between radar and radiometer observations.

Keywords: $\quad$ Downscaling, Brightness temperature, Radar backscatter, SMAP, SMAPEx 
Wu et al., Downscaling of coarse-resolution radiometer brightness temperature by high-resolution radar backscatter

\section{INTRODUCTION}

Soil moisture is of great importance to global water cycle monitoring and prediction, especially in agriculture, hydrology and meteorology (Wagner et al. 2003). With the development of remote sensing technology (Schmugge et al. 2002), soil moisture mapping over large areas by remote sensing is becoming a potential alternative to traditional monitoring by in situ networks of stations. Consequently, methods are being developed to make use of this emerging soil moisture information to constrain numerical model prediction of soil moisture (Shi et al. 2009), and hence improve the forecasting of weather and floods, leading to significant national benefit.

Over the past decade, passive microwave remote sensing techniques for soil moisture mapping have been accepted as the preferred approach, due to the stronger and more direct connection between the observed brightness temperature $(\mathrm{Tb})$ and the near surface soil moisture $(\sim 5 \mathrm{~cm})$, than with active microwave sensing (radar backscatter) or visible/thermal/infrared data (Kerr 2007). Despite the strong soil moisture relationship of the passive microwave radiometer approach, it suffers from having a relatively low spatial resolution, which is on the order of $36 \mathrm{~km}$ at L-band. Conversely, active microwave observations have a much higher spatial resolution (better than $1 \mathrm{~km}$ ), but with a more convoluted relationship to changes in soil moisture due to the confounding effects of vegetation and surface roughness. Consequently, NASA is developing the Soil Moisture Active Passive (SMAP) mission (Entekhabi et al. 2010), scheduled to be launched in late 2014, to take advantage of the synergy between the two approaches. The rationale behind SMAP is to use the spatial information in the active observations within a downscaling algorithm to provide a downscaled brightness temperature product at $9 \mathrm{~km}$ resolution, which is subsequently converted to soil moisture using a passive microwave retrieval algorithm.

The proposed downscaling algorithm to be applied in the SMAP mission is based on the near-linear relationship between radar backscatter $\sigma$ and radiometer brightness temperature $T b$. Such a relationship has been observed using data collected from the airborne Passive and Active L-band System (PALS) instrument during the Soil Moisture Experiments 2002 and the Soil Moisture Active Passive Validation Experiment 2008 (Das et al. 2011, Das et al. 2013). However, tests of the SMAP baseline downscaling algorithm are limited, and only a few studies have used experimental data. Therefore, the objectives of this paper mainly involve: i) to evaluate the effectiveness of this linear brightness temperature downscaling method, using experimental passive and active observations collected from SMAPEx field campaigns in Australia; and ii) to assess the capability of this algorithm using high-resolution data, including $1 \mathrm{~km}$ resolution brightness temperature and $10 \mathrm{~m}$ backscatter. In this study, the $1 \mathrm{~km}$ resolution radiometer data are disaggregated to $250 \mathrm{~m}$ resolution by radar observations, keeping the same ratio of the SMAP mission (from $36 \mathrm{~km}$ to $9 \mathrm{~km}$ ), thus evaluating the performance of this downscaling algorithm under a homogeneous and heterogeneous land cover conditions.

\section{STUDY SITE}

The SMAPEx study site (see Figure 1a) is located in the Murrumbidgee catchment near the township of Yanco $\left(-34.67^{\circ} \mathrm{N},-35.01^{\circ} \mathrm{N}, 145.97^{\circ} \mathrm{E}, 146.36^{\circ} \mathrm{E}\right)$. The site has been chosen for testing the SMAP algorithm performance due to its flat topography, dense monitoring station network, and spatial variability in soil, vegetation and land use (Panciera et al. 2013). The SMAPEx experimental design aimed to simulate data from the SMAP mission over a landscape typical of south-eastern Australia. To that end airborne data were collected over an area the size of a SMAP radiometer footprint (approximately $36 \mathrm{~km} \times 38 \mathrm{~km}$ at this latitude) and two additional focus areas using a SMAP simulator. The airborne SMAP simulator allows high spatial resolution active and passive microwave remote sensing measurements to be made at L-band, with characteristics similar to those expected from SMAP. The facility includes the Polarimetric L-band Multibeam Radiometer (PLMR) and the Polarimetric L-band Imaging Synthetic aperture radar (PLIS) which, when used together on the same aircraft, provide a SMAP-like data set useful for developing and testing of the algorithms applicable to the SMAP mission viewing configuration.

Data used in this study were collected from the second field campaign SMAPEx-2 from $6^{\text {th }}$ to $10^{\text {th }}$ July 2010 , which included 3 days of Regional flights over the entire SMAPEx area and 2 days of Target flights, each conducted over a focus area ("YA" and "YB", see Figure 1a). While the YA area is dominated by crops with variations in vegetation characteristics and land conditions, the YB area is a grassland site with relatively homogenous conditions. Data collected from Regional flights included $1 \mathrm{~km}$ resolution PLMR $T b$ and $10 \mathrm{~m}$ resolution PLIS $\sigma$, which in this study were used to analyse the relationship between $T b$ and $\sigma$; data obtained from the Target flights included $100 \mathrm{~m}$ resolution PLMR $T b$ and $10 \mathrm{~m}$ resolution PLIS $\sigma$ which were used to perform the $T b$ downscaling from $1 \mathrm{~km}$ to $250 \mathrm{~m}$ resolution. 
Wu et al., Downscaling of coarse-resolution radiometer brightness temperature by high-resolution radar backscatter

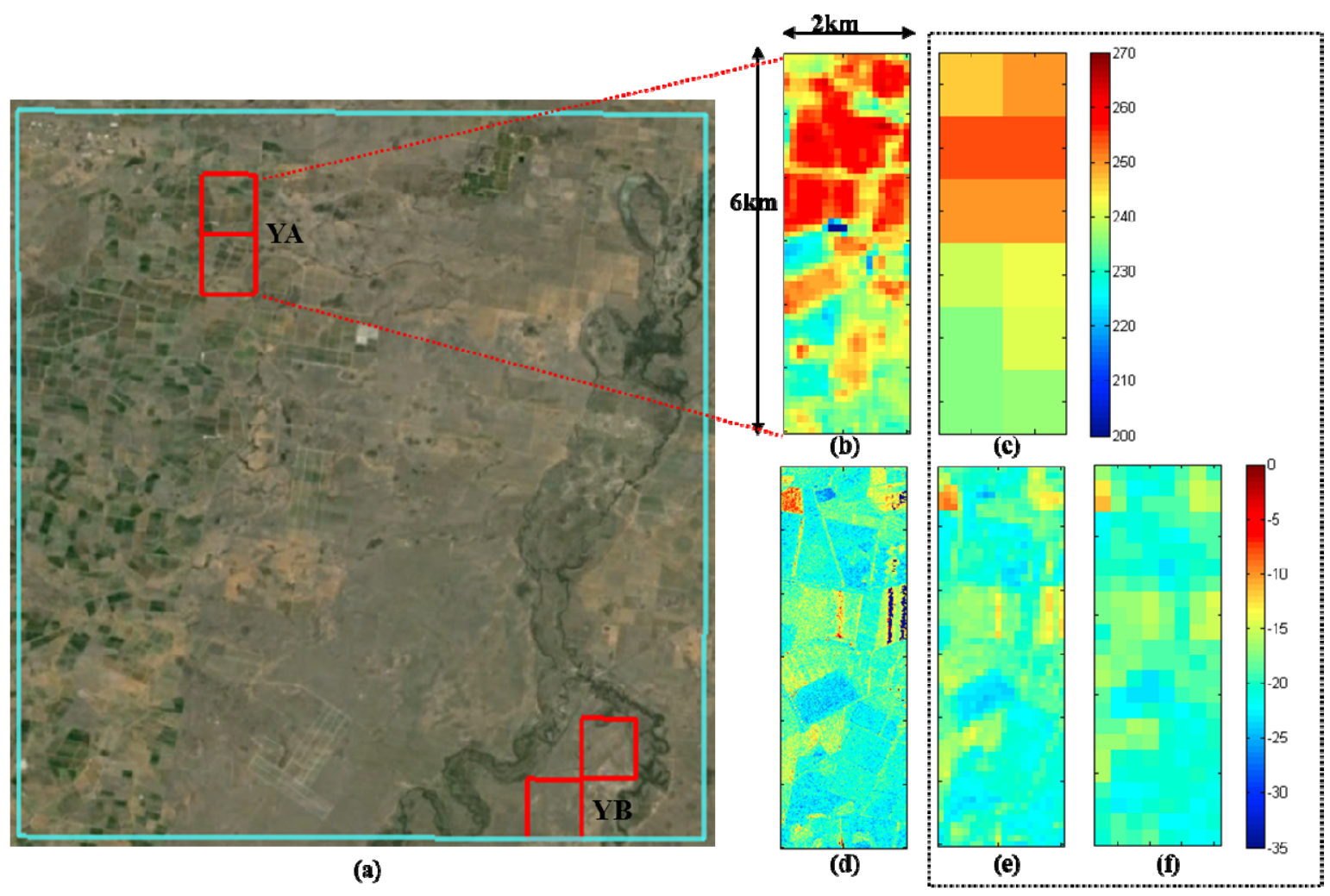

Figure 1. (a) Overview of SMAPEx study site $(38 \mathrm{~km} \times 36 \mathrm{~km}$ size $)$ and target areas YA and YB which are used to test the downscaling algorithm; (b) PLMR radiometer brightness temperatures (Tb) over the YA target area at $v$-polarization and $100 \mathrm{~m}$ resolution, and (c) aggregated to $1 \mathrm{~km}$ resolution; (d) observed $10 \mathrm{~m}$ resolution PLIS radar backscatters $(\sigma)$ over the YA area at $v v$-polarization, aggregated to (e) $100 \mathrm{~m}$ resolution and (f) $250 \mathrm{~m}$ resolution, respectively.

In order to closely replicate the viewing configuration of SMAP, both the PLMR and PLIS data were normalized for incidence angle variation to the constant $40^{\circ}$ angle of SMAP, using a Cumulative Distribution Function based method (Ye et al. 2013). The error of this normalization method for PLMR is $2.4 \mathrm{Kelvin}(\mathrm{K})$ at $1 \mathrm{~km}$ resolution; for PLIS it is $3.3 \mathrm{~dB}$ at $10 \mathrm{~m}$ resolution and $1.7 \mathrm{~dB}$ when aggregated to $100 \mathrm{~m}$ resolution.

The observed $100 \mathrm{~m}$ resolution PLMR data from Target flights were linearly aggregated to $1 \mathrm{~km}$ resolution, and the observed $10 \mathrm{~m}$ resolution PLIS data aggregated to $100 \mathrm{~m}$ and $250 \mathrm{~m}$ in order to evaluate the downscaling algorithm at different resolutions. An example of the aggregated data over YA area is shown in Figure 1.

\section{DOWNSCALING METHOD}

The baseline downscaling algorithm proposed for SMAP is based on the assumption of a near-linear relationship between L-band brightness temperatures (in the unit of Kelvin) and L-band backscatters (in the unit of Decibel) observed at the same spatial resolution (ATBD 2012, Das et al. 2011, Das et al. 2013). In the following the naming convention of ' $C$ ' (coarse), and ' $F$ ' (fine) represents the brightness temperature $T b$ $(1 \mathrm{~km})$ and backscatter $\sigma(250 \mathrm{~m})$ resolutions, respectively. Implementation of this method first requires a linear regression of observations to derive the coefficients of the relationship:

$$
T b_{p}(C)=\alpha(C)+\beta(C) \times \sigma_{p p}(C),
$$

where $p$ indicates the radiometer polarization, including $h$ - and $v$-pol; $p p$ means co-polarization of radar observations $\sigma$, including $h h$ or $v v$-pol. Correlations between four different combinations of $T b_{p}$ and $\sigma_{p p}$ are analysed in the next section. The value for $\sigma_{p p}(C)$ is obtained by aggregating $10-30 \mathrm{~m}$ resolution PLIS data within the coarse footprint $C$ having $T b_{p}(C)$ using the $1 \mathrm{~km}$ resolution PLMR observations. The method further assumes that parameters $\alpha(C)$ and $\beta(C)$, which depend on vegetation cover and type as well as surface roughness, are time-invariant and homogenous over the coarse pixel, so that the $\alpha(C)$ and $\beta(C)$ derived at $C$ resolution can be applied at $F$ resolution. By virtue of this assumption, downscaling of $T b$ to one grid cell $F$ can be achieved by writing (1) as 
Wu et al., Downscaling of coarse-resolution radiometer brightness temperature by high-resolution radar backscatter

$$
\operatorname{Tbp}(F j)=\alpha(F j)+\beta(F j) \times \sigma p p(F j),
$$

where $\operatorname{Tb}_{p}\left(F_{j}\right)$ is the brightness temperature value at spatial scale $F$ within a particular pixel of resolution $C$, and $\sigma_{p p}\left(F_{j}\right)$ is the corresponding radar backscatter value. By averaging both sides of (2), one obtains

$$
<\operatorname{Tbp}(F j)>=<\alpha(F j)>+<\beta(F j)>\times<\sigma p p(F j)>.
$$

Here $<>$ is used to indicate averaging across $C$, which yields $\left\langle T b_{p}\left(F_{j}\right)>=T b_{p}(C)\right.$, as each $F$-resolution pixel within $C$ shares the same value of $T b$ at that scale. Subtracting (3) from (2), and considering $\alpha$ and $\beta$ as homogeneous and therefore equal at each scale, the downscaled $T b$ at scale $F$ can be obtained as

$$
\operatorname{Tbp}(F j)=\operatorname{Tbp}(C)+\beta(C) \times[\sigma p p(F j)-\sigma p p(C)] .
$$

Given that the vegetation conditions and surface characteristics across the entire study area are quite varied, it would result in a relatively poor performance of the baseline downscaling algorithm due to the influence of variable vegetation and roughness patterns on parameter $\beta$. However, since the radar also provides highresolution cross-polarization ( $h v$-pol) backscatter measurements at scale $F$, which are sensitive to vegetation and surface roughness characteristics, a further hypothesis is that the sub-grid heterogeneity of parameter $\beta$ within scale $C$ can be corrected using the difference $\left[\sigma_{p q}(C)-\sigma_{p q}\left(F_{j}\right)\right]$ from the radar, where $p q$ represents $h v$ pol. This heterogeneity indicator can be converted to variations in co-polarization $p p$ backscatter by multiplying a sensitivity parameter $\left[\delta \sigma_{p p}\left(F_{j}\right) / \delta \sigma_{p q}\left(F_{j}\right)\right]_{C}$, denoted by the variable $\gamma$ of the particular grid cell $C$ for the time of year: $\gamma=\left[\delta \sigma_{p p}\left(F_{j}\right) / \delta \sigma_{p q}\left(F_{j}\right)\right]_{C}$. In other words, this new term $\gamma \times\left[\sigma_{p q}(\mathrm{C})-\sigma_{p q}\left(\mathrm{~F}_{\mathrm{j}}\right)\right]$ can be described as the projection of the cross-polarization sub-grid heterogeneity onto the co-polarization space, thus converting the information of vegetation and surface characteristics to the variation of co-polarized backscatter. This term is converted to $T b$ through the multiplication by $\beta(C)$ in (4). Consequently, taking into account the vegetation conditions and surface characteristics, downscaled $T b$ at $F$ scale is obtained by

$$
\operatorname{Tbp}(F j)=T b p(C)+\beta(C) \times\{[\sigma p p(F j)-\sigma p p(C)]+\gamma \times[\sigma p q(C)-\sigma p q(F j)]\} .
$$

Using (5) the downscaled $T b$ is obtained for each pixel in the YA or YB area at 250m resolution; other scale resolutions such as $100 \mathrm{~m}$ can be obtained by using $100 \mathrm{~m}$ resolution PLIS data, instead of $250 \mathrm{~m}$ resolution, as the input of the fine resolution PLIS data in equation (5). This study downscales the $1 \mathrm{~km}$ resolution $T b$ to $250 \mathrm{~m}$ resolution, to test the ability of this baseline downscaling algorithm at the same resolution ratio as SMAP, which aims to downscale $36 \mathrm{~km}$ resolution $T b$ observations to $9 \mathrm{~km}$. Furthermore, the downscaling algorithm is applied with a $100 \mathrm{~m}$ target downscaled resolution to evaluate the performance of the downscaling approach at different scales. The downscaled $T b$ at fine resolution (including $100 \mathrm{~m}$ and $250 \mathrm{~m}$ ) is heavily dependent on the quality of the overall PLMR $T b$ at each $1 \mathrm{~km}$ by $1 \mathrm{~km}$ pixel, the relative backscatter difference within the coarse grid $(1 \mathrm{~km})$, and the relationship with $T b$ as represented by the regression slope that adds to the background value $T b_{p}(C)$. The downscaled results at different resolutions are evaluated by comparing with PLMR $T b$ data at the original resolution of $100 \mathrm{~m}$ and $250 \mathrm{~m}$ respectively.

\section{RESULTS AND DISCUSSION}

\subsection{Estimation of $\beta$ and $\gamma$}

Prior to carrying out the downscaling algorithm, the relationship between $T b$ at both polarizations ( $h$ and $v$ ) and $\sigma$ at two polarizations ( $h h$ and $v v$ ) was determined to verify the linear relationship assumption which is the foundation of the approach, and to estimate parameter $\beta$ to be used in Eq. (5). The parameter $\gamma$ also has to be estimated to represent the sensitivity of $\sigma_{v v}$ to $\sigma_{h v}$.

As described, this baseline downscaling algorithm is based on the assumption that brightness temperature $T b_{p}$ is linearly related to the backscatter $\sigma_{p p}$ at the same scale. Therefore, the robustness of this linear relationship is tested in this section using the four different combinations of $T b_{p}$ and $\sigma_{p p}$. The aim is to determine the best combination of radiometer and radar channels for estimating parameter $\beta$. In this case, data from the
Table 1: Relationship between $T b(K)$ and $\sigma(d B)$ at different polarizations over the YA and YB areas, $\beta$ is the regression slope of $T b$ and $\sigma$, while $R$ is the correlation coefficient between $T b$ and $\sigma$. A total of 36 pairs $T b$ and $\sigma$ were used to estimate $\beta$, with standard error (in bracket) across each area.

\begin{tabular}{|c|c|c|c|c|}
\hline \multirow{2}{*}{} & \multicolumn{2}{|c|}{ YA (crops) } & \multicolumn{2}{c|}{ YB (grassland) } \\
\cline { 2 - 5 } & $T b_{h}$ & $T b_{v}$ & $T b_{h}$ & \multicolumn{1}{c|}{$T b_{v}$} \\
\hline \multirow{2}{*}{$\sigma_{v v}$} & $\beta:-4.3(1.2)$ & $\beta:-3.2(1.0)$ & $\beta:-3.0(1.0)$ & $\beta:-2.3(0.8)$ \\
\cline { 2 - 5 } & $R: 0.62$ & $R: 0.65$ & $R: 0.72$ & $R: 0.80$ \\
\hline \multirow{3}{*}{$\sigma_{h h}$} & $\beta:-5.2(1.3)$ & $\beta:-3.8(0.9)$ & $\beta:-4.5(0.9)$ & $\beta:-3.3(0.7)$ \\
\cline { 2 - 5 } & $R: 0.59$ & $R: 0.61$ & $R: 0.65$ & $R: 0.71$ \\
\hline \multirow{2}{*}{$\sigma_{h v}$} & $\beta:-5.1(1.2)$ & $\beta:-4.1(1.2)$ & $\beta:-4.7(1.0)$ & $\beta:-3.0(0.9)$ \\
\cline { 2 - 5 } & $R: 0.21$ & $R: 0.28$ & $R: 0.34$ & $R: 0.37$ \\
\hline
\end{tabular}


Wu et al., Downscaling of coarse-resolution radiometer brightness temperature by high-resolution radar backscatter

three Regional flights were used. Parameter $\beta$ was calculated using the regression between the PLMR $T b$ (observed at $1 \mathrm{~km}$ ) and the PLIS $\sigma$ (aggregated to $1 \mathrm{~km}$ ) collected over each $1 \mathrm{~km}$ pixel in YA or YB area. Two different values of parameter $\beta$ were estimated: one value for the YA area (characterizing the sensitivity of $T b_{p}$ to $\sigma_{p p}$ over crops), and a second value for the YB area (characterizing the sensitivity over grassland). The average $\beta$ across each area, obtained from 3 days' time-series over 12 pixels within this area, together with its standard error (around 1.0K/dB for different polarization), are listed in Table 1. The $\sigma$ at $v v$-polarization had the best correlation to $T b$, with correlation coefficient $R=0.65$ and 0.81 for crops and grassland respectively. Conversely, $\sigma$ at $h h$-polarization was less correlated to $T b$, and showed little correlation at $h v$ polarization $(R=0.28$ and 0.37$)$. Therefore, $\sigma$ at $v v$-polarization and at $h h$-polarization will be used to downscale PLMR $T b$ to further confirm the influence of the correlation between $T b$ and $\sigma$ at different polarization on the performance of the downscaling algorithm. Moreover, the magnitudes of $\beta$ values over the YA area are larger than those over the YB area, indicating that the sensitivity of $T b$ to $\sigma$ is stronger over crops than over grassland. Or in other words, the sensitivity of $\sigma$ to $T b$ is stronger for grassland. This is mainly because radar backscatter is more sensitive to vegetation conditions than the $T b$, which results in poorer correlation between $\sigma$ and $T b$ in the YA area than in YB area. The availability of only 3 days' timeseries will influence the robustness of $\beta$ estimation and further influence the accuracy of downscaling.

The parameter $\gamma$ was estimated using the pairs of $\sigma_{v v}$ and $\sigma_{h v}$ within each $1 \mathrm{~km}$ pixel, so as to obtain the regression slope $\gamma$ at each $1 \mathrm{~km}$ pixel. Consequently, $\gamma$ varies across the entire YA or YB area, with a range from 0.1 to 0.5 for both areas.

\subsection{Downscaled results}

Based on the respective value of $\beta$ and $\gamma$ matrix over YA and YB, the $1 \mathrm{~km} T b$ aggregated from Target flights were downscaled to $100 \mathrm{~m}$ and $250 \mathrm{~m}$ resolution, by using PLIS $\sigma$ at $100 \mathrm{~m}$ and $250 \mathrm{~m}$ resolution (aggregated from $10 \mathrm{~m}$ resolution) respectively. The downscaled $T b$ were then compared with the reference $T b$ directly measured from PLMR at $100 \mathrm{~m}$ and $250 \mathrm{~m}$ resolution in order to evaluate the accuracy of the downscaling algorithm. Figure 2 and Figure 3 show the downscaled $v$-polarized $T b$ and the difference between downscaled and reference $T b$ at different resolutions over YA and YB area respectively. By comparing the differences over $\mathrm{YA}$ and $\mathrm{YB}$, it is noted that results over the $\mathrm{YB}$ area show an overall smaller error than over YA, which could be attributed to the influence of heterogeneous vegetation in the YA area on the accuracy of the downscaling algorithm. Quantitative details are displayed in Table 2, from which it can be seen that the RMSE of YB has an improvement of approximately $3 K$ at $h$ polarization and $2.5 \mathrm{~K}$ at $v$-polarization over $\mathrm{YA}$, confirming the results from Figure 2 and Figure 3. In addition, results at $v$-polarization are better than those at $h$-polarization for both the YA and YB area, with an improvement of around $2 K$. This is due to $\sigma_{v v}$ being more strongly related to $T b$ at $v$-polarization than $T b$ at $h$-polarization during the estimation of $\beta$. Moreover, the results at $250 \mathrm{~m}$ resolution were more accurate than those at $100 \mathrm{~m}$, on the order of $1 K$ improvement in terms of RMSE. This is possibly because the speckle noise of radar backscatter is reduced when upscaling from $100 \mathrm{~m}$ to $250 \mathrm{~m}$ resolution, and the error of incidence angle normalization is reduced from $100 \mathrm{~m}$ to $250 \mathrm{~m}$ resolution.

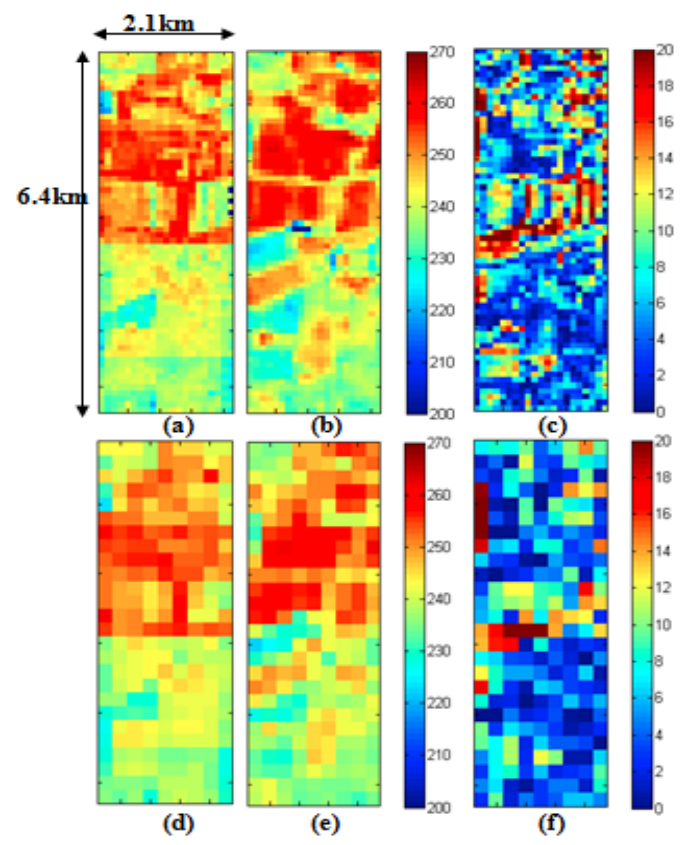

Figure 2. Evaluation of the downscaling algorithm at vpolarization over the YA area: (a) Downscaled $T b$ at $100 \mathrm{~m}$ resolution; (b) Reference $T b$ at $100 \mathrm{~m}$ resolution and (c) the difference; (d) Downscaled $T b$ at $250 \mathrm{~m}$ resolution; (e) Reference $T b$ at $250 \mathrm{~m}$ resolution; and (c) the difference.

Table 2: RMSE $(K)$ of downscaling algorithm using $\sigma_{v v}$ and $\sigma_{h h}$ (in bracket) at different polarizations and at different resolutions $(100 \mathrm{~m}$ and $250 \mathrm{~m})$ over YA and YB areas.

\begin{tabular}{|c|c|c|c|c|}
\hline \multirow{2}{*}{} & \multicolumn{2}{|c|}{ YA (crops) } & \multicolumn{2}{c|}{ YB (grassland) } \\
\cline { 2 - 5 } & $h$-pol & $v$-pol & $h$-pol & $v$-pol \\
\hline $100 \mathrm{~m}$ & $10.2(12.7)$ & $8.6(10.1)$ & $7.5(8.3)$ & $5.7(6.6)$ \\
\hline $250 \mathrm{~m}$ & $9.0(11.1)$ & $7.1(8.8)$ & $5.8(6.9)$ & $4.6(5.5)$ \\
\hline
\end{tabular}




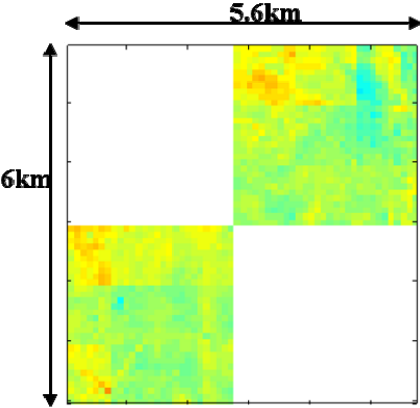

(a)

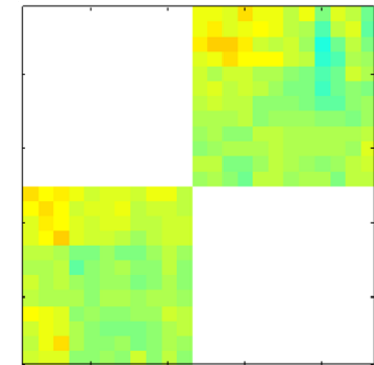

(d)

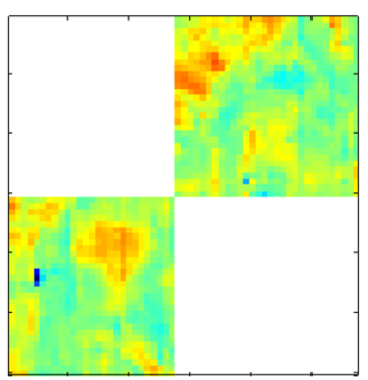

(b)

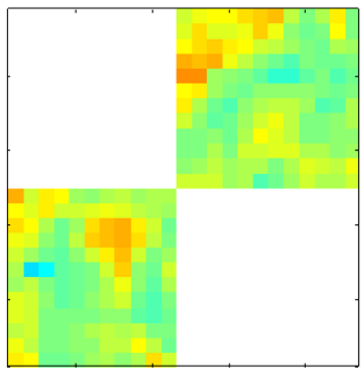

(e)
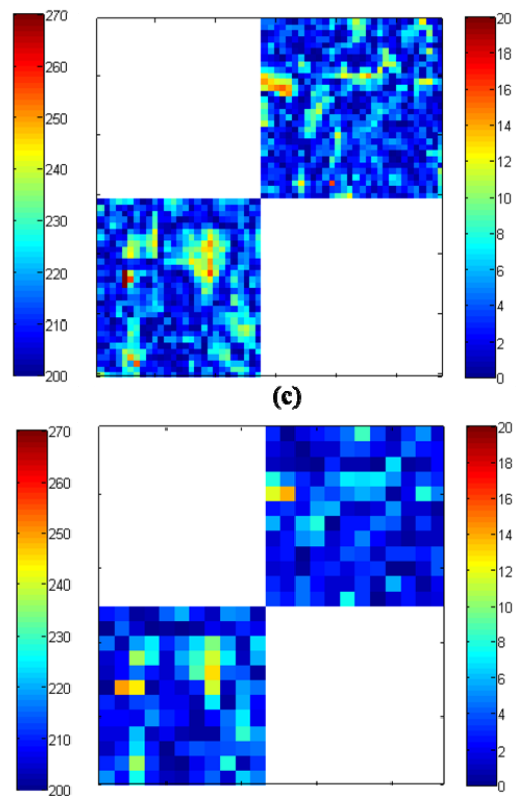

(f)

Figure 3. Evaluation of the downscaling algorithm at v-polarization over the YB area: (a) Downscaled $T b$ at $100 \mathrm{~m}$ resolution; (b) Reference $T b$ at $100 \mathrm{~m}$ resolution and (c) the difference; (d) Downscaled $T b$ at $250 \mathrm{~m}$ resolution; (e) Reference $T b$ at $250 \mathrm{~m}$ resolution; and (c) the difference.

Additionally, the estimation of $\beta$ at $1 \mathrm{~km}$ resolution is closer to $\beta$ at $250 \mathrm{~m}$ than at $100 \mathrm{~m}$ resolution. It is noted that downscaled results based on $\sigma_{h h}$ resulted in an RMSE on the order of $\sim 1.8 \mathrm{~K}$ greater than when using $\sigma_{v v}$, confirming the conclusion from Table 1 that $\sigma_{v v}$ is better correlated with $T b$. The influence from the variation in $\beta$ (as indicated by the standard error in Table 1) was also analyzed which was found to result in approximately $2 K$ error in the downscaled $T b$ at $100 \mathrm{~m}$ resolution. Therefore, a better estimation of $\beta$ can be expected from longer time-series observations over each $1 \mathrm{~km}$ pixel, thus improving the downscaling performance.

To further evaluate the skill of the downscaling algorithm, the correlation between downscaled $T b$ and the reference $T b$ was studied with respect to different land cover at $100 \mathrm{~m}$ and $250 \mathrm{~m}$ resolution, with results displayed in Figure 4. While the black line represents a RMSE between downscaled and reference $T b$ of $0 K$, the dashed line represents RMSE of $\pm 4 K$. It is noted from Figure 4 that the variation of $T b$ over the YA area is much larger than the $\mathrm{YB}$ area, in response to the wider range of vegetation and land cover across the cropping area YA than the relatively homogenous area YB. In addition, a greater fraction of the data at $100 \mathrm{~m}$ resolution is outside the $4 K$ error range than at $250 \mathrm{~m}$ resolution, due to the reasons stated above. It is also noted that a greater fraction of the data fell within the $4 K$ range over the $\mathrm{YB}$ area than for the YA area. Thus, it can be noted that the downscaling algorithm has an overall better performance over grassland than the cropping area.

The target error of downscaled $T b$ at $9 \mathrm{~km}$ resolution of the SMAP mission is around $2.4 \mathrm{~K}$ for vegetation water content less than $5 \mathrm{~kg} / \mathrm{m}^{2}$, which is much lower than that achieved here. The reasons for larger errors when using the downscaling algorithm in this study may include: i) the availability of only 3 days of Regional flights for estimating parameter $\beta$, as the accuracy of $\beta$ estimation influences the resulting accuracy of downscaling, which is expected to be improved when using a longer time series of

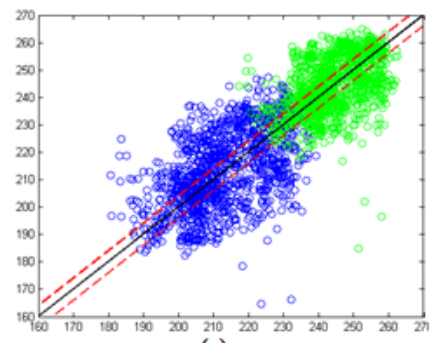

(a)
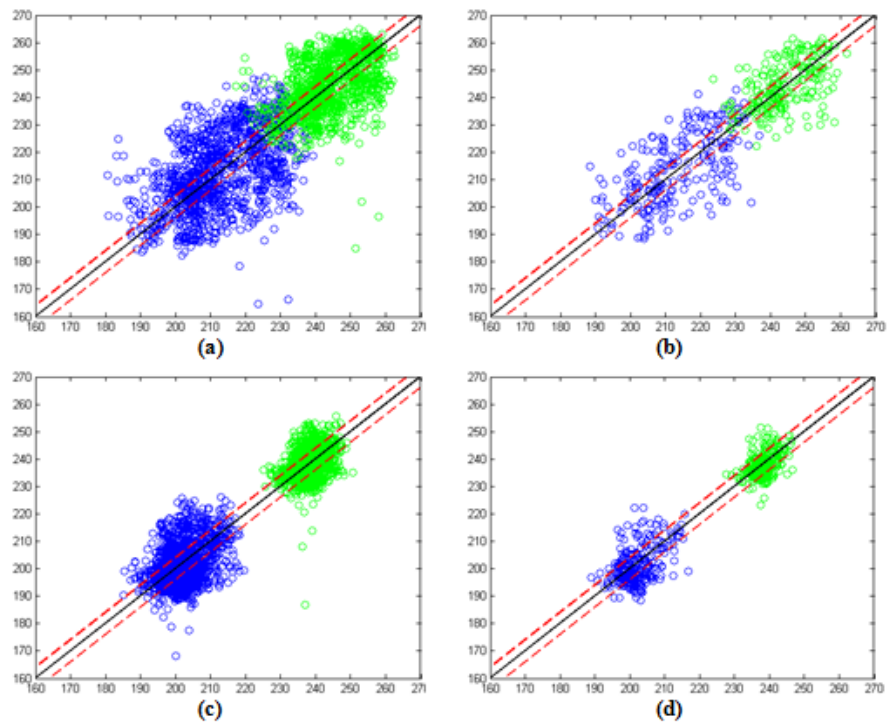

(d)

Figure 4: Agreement between downscaled $T b$ (horizontal) and reference $T b$ (vertical) at $v$-polarization: (a) at $100 \mathrm{~m}$ resolution over YA area; (b) at $250 \mathrm{~m}$ resolution over YA area; (c) at $100 \mathrm{~m}$ resolution over YB area; and (d) at $250 \mathrm{~m}$ resolution over YB area. 
Wu et al., Downscaling of coarse-resolution radiometer brightness temperature by high-resolution radar backscatter

data; ii) the incidence angle normalization prior to the downscaling algorithm induced an error around $1.5 \mathrm{~dB}$ for PLIS at $250 \mathrm{~m}$ resolution and around $2.4 K$ for PLMR at $1 \mathrm{~km}$ resolution.

\section{CONCLUSION}

The objective of this study was to test the baseline downscaling approach for the SMAP mission, using high resolution data from SMAPEx campaign in Australia. In particular, the aim was to constrast the algorithm results from an area of low heterogeneity with those from a more typical level of heterogeneity, and to assess the best polarization combinations to be used. In this study, radar backscatter aggregated to $100 \mathrm{~m}$ and $250 \mathrm{~m}$ resolution were used to downscale radiometer brightness temperature at $1 \mathrm{~km}$ resolution to $100 \mathrm{~m}$ and $250 \mathrm{~m}$ resolution respectively. The results showed that the RMSE of downscaled $T b$ at $v$-polarization was better than $h$-polarization, with an improvement of around $2 K$, due to $T b$ at $v$-polarization being more strongly corelated to $\sigma_{v v}$ than $T b$ at $h$-polarization. Downscaling to $250 \mathrm{~m}$ resolution resulted in an RMSE improvement of approximately $1 K$ over downscaling at $100 \mathrm{~m}$. In terms of the performance over different land covers, it is found that RMSE of the downscaling result over relatively homogenous grassland has an improvement of $3 \mathrm{~K}$ when compared to the heterogeneous cropping area. It is shown that the accuracy of the downscaling approach is primarily determined by the heterogeneity of vegetation characteristics across the study area, as well as the sensitivity of brightness temperature to radar backscatter, as reflected in the parameter $\beta$. Based on the standard error of the $\beta$ in this study, a more robust $\beta$ is expected using a longer time series of observations over each $1 \mathrm{~km}$ pixel in order to distinguish the surface conditions across the entire area, thus resulting in more accurate downscaling results, but the main conclusions are not expected to change. It is also important that future studies will more extensively investigate the estimation of $\beta$ and $\gamma$ with respect to crop types, vegetation water content and surface roughness, with the aim to improve the accuracy of downscaling results.

\section{ACKNOWLEDGMENTS}

The SMAPEx field campaigns and related research development have been funded by the Australian Research Council (DP0984586, LE0453434 and LE0882509). The authors acknowledge the collaboration of a large number of scientists from throughout Australia and around the world, which provided significant contribution to the campaign's design and execution. The authors also acknowledge the scholarship awarded by Monash University to support Xiaoling Wu's $\mathrm{PhD}$ research.

\section{REFERENCES}

ATBD: Algorithm Theoretical Basis Document, L2 \& L3 Radar/Radiometer Soil Moisture (Active/Passive) Data Products, Initial Release, v.1, 2012. http://smap.jpl.nasa.gov/science/dataproducts/ATBD/

DAS, N. N., D. Entekhabi, E. G. Njoku. (2011). An algorithm for merging SMAP radiometer and radar data for high-resolution soil moisture retrieval. IEEE Transactions on Geoscience and Remote Sensing, 49(5), 1504-1512.

Das, N. N., D. Entekhabi, E. G. Njoku, et al. (2013). Tests of the SMAP Combined Radar and Radiometer Algorithm Using Airborne Field Campaign Observations and Simulated Data. IEEE Transaction on Geoscience and Remote Sensing, In Press

Entekhabi, D., E. G. Njoku, et al. (2010). The Soil Moisture Active Passive (SMAP) Mission. Proceedings of the IEEE, 98(5), 704-716.

Kerr, Y. (2007). Soil moisture from space: Where are we? Hydrogeology Journal, 15(1), 117-120.

Panciera, R., Walker, J. P., Jackson, T. J., et al. (2013). The Soil Moisture Active Passive Experiments (SMAPEx): Towards Soil Moisture Retrieval from the SMAP Mission. IEEE Transaction on Geoscience and Remote Sensing, PP(99), 1-18.

Schmugge, T. J., W. P. Kustas, J. C. Ritchie, et al. (2002). Remote sensing in hydrology. Advances in Waster Resources 25, 1367-1385.

Shi, X. K., J. Wen, et al. (2009). Application of satellite microwave remote sensed brightness temperature in the regional soil moisture simulation. Hydrol. Earth Syst. Sci. Discuss, 6(1), 1233-1260.

Wagner, W., Scipal, K., Pathe, C., et al. (2003). Evaluation of the agreement between the first global remotely sensed soil moisture data with model and precipitation data. J. Geophys. Res., 108, 4611.

Ye, N., Walker, J. P. \& Rudiger, C. (2013). A cumulative distribution function method for normalising multiangle microwave observations. IEEE Transaction on Geoscience and Remote Sensing, In Review. 